\title{
Outcome for triple negative breast cancer in a retrospective cohort with an emphasis on response to platinum-based neoadjuvant therapy
}

\author{
Elaine M. Walsh ${ }^{1,2} \cdot$ Aliaa Shalaby ${ }^{1} \cdot$ Mark O'Loughlin $^{1} \cdot$ Nessa Keane ${ }^{1} \cdot$ Mark J Webber $^{1} \cdot$ Michael J. Kerin $^{3}$. \\ Maccon M. Keane ${ }^{2}$. Sharon A. Glynn ${ }^{1}$. Grace M. Callagy ${ }^{1}$ (D)
}

Received: 29 May 2018 / Accepted: 19 November 2018 / Published online: 28 November 2018

(C) The Author(s) 2018

\begin{abstract}
Purpose The rate of pathological complete response (pCR) for patients with triple negative breast cancer (TNBC) is increased when carboplatin is added to neo-adjuvant chemotherapy (NACT). However, while phase III trial data showing a survival benefit are awaited, carboplatin is not yet standard-of-care for TNBC. The aim of this study was to examine the rate of pCR and the outcome for those treated with carboplatin and to examine the predictors of response to therapy.

Methods The retrospective series comprised 333 consecutive patients with TNBC (median follow-up time, 43 months). Adjuvant chemotherapy was given to $51 \%(n=168)$ of patients and $29 \%(n=97)$ received anthracycline-taxane NACT with carboplatin given to $9 \%(n=31)$ of patients.

Results Overall, $25 \%(n=78)$ of patients experienced a breast cancer recurrence and $22 \%(n=68)$ died from disease. A pCR breast and pCR breast/axilla was more common in those who received carboplatin $(n=18,58 \%$ and $n=17,55 \%$, respectively) compared those who did not $(n=23,36 \%$ and $n=18,28 \%$, respectively) ( $p=0.041$ and $p=0.011$, respectively). By multivariable analysis, carboplatin and high tumor grade were independent predictors of pCR breast/axilla $\left(\mathrm{OR} \mathrm{non}_{\mathrm{pCR}}=0.17\right.$; 95\% CI 0.06-0.54; $p=0.002$; and $\mathrm{OR}_{\text {non-pCR }}=0.05,95 \%$ CI $0.01-0.27 ; p<0.001$, respectively). pCR breast/axilla was an independent predictor of DFS $\left(\mathrm{HR}_{\text {non-pCR }}=6.23 ; 95 \%\right.$ CI 1.36-28.50; $\left.p=0.018\right)$, metastasis-free survival $\left(\mathrm{HR}_{\text {non-pCR }}=5.08\right.$; 95\% CI 1.09-23.65; $p=0.038)$ and BCSS ( HR $_{\text {non-pCR }}=8.52 ; 95 \%$ CI $\left.1.09-66.64 ; p=0.041\right)$.

Conclusion Carboplatin therapy and high tumor grade are associated with a significant increase in the rate of pCR, which is an independent predictor of outcome. These data support the use of carboplatin in NACT for TNBC, while results from phase III studies are awaited.
\end{abstract}

Keywords Triple negative $\cdot$ Breast cancer $\cdot$ Survival $\cdot$ Pathological complete response $\cdot$ Neoadjuvant $\cdot$ Carboplatin

\begin{tabular}{llll}
\multicolumn{2}{l}{ Abbreviations } & DFS & Disease-free survival \\
AUC & Area under the curve & EGFR & Epidermal growth factor receptor \\
BCSS & Breast cancer-specific survival & ER & Estrogen receptor \\
BCT & Breast conserving therapy & FISH & Fluorescent in situ hybridization \\
BRCA1/2 & Breast cancer gene 1/2 & GUH & Galway University Hospital \\
CALGB & Cancer and Leukemia Group B & HER2 & Human epidermal growth factor receptor 2 \\
CI & Confidence interval & H\&E & Hematoxylin and eosin stain \\
CK & Cytokeratin & HR & Hazard ratio \\
& IHC & Immunohistochemistry \\
\hline $\begin{array}{l}\text { Electronic supplementary material The online version of this } \\
\text { article (https://doi.org/10.1007/s10549-018-5066-6) contains }\end{array}$ & M1 & Metastatic disease \\
$\begin{array}{l}\text { supplementary material, which is available to authorized users. } \\
\text { Mrace M. Callagy }\end{array}$ & MFS & Metastasis-free survival \\
$\begin{array}{l}\text { grace.callagy @ nuigalway.ie } \\
\text { Extended author information available on the last page of the article }\end{array}$ & NSABP & Neoadjuvant chemotherapy \\
National Surgical Adjuvant Breast and Bowel
\end{tabular}




$\begin{array}{ll}\text { OS } & \text { Overall survival } \\ \text { PARP } & \text { Poly-ADP-ribose-polymerase } \\ \text { pCR } & \text { Pathological complete response } \\ \text { PR } & \text { Progesterone receptor } \\ \text { TMA } & \text { Tissue microarray } \\ \text { TNBC } & \text { Triple negative breast cancer }\end{array}$

\section{Background}

Triple negative breast cancer (TNBC) is an operational term used to define tumors that lack expression of estrogen receptor (ER), progesterone receptor (PR) and lack amplification or overexpression of human epidermal growth factor receptor 2 (HER2) [1, 2]. Approximately 20\% of breast cancers are TNBC [3]. A small proportion of TNBCs, including adenoid cystic, secretory, adenosquamous and carcinoma, have low aggressive potential and follow an indolent course. However, the majority of TNBCs, most of which are invasive ductal carcinomas, are associated with high rates of early distant recurrence and short survival times compared to other breast cancer subtypes [1, 2, 4-9]. Almost all women with metastatic TNBC will die of their disease within 5 years of diagnosis $[1,3,10,11]$; however, those who remain disease-free 8 years after initial diagnosis rarely die of breast cancer [1, 2, 4], unlike other breast cancer subtypes.

A subgroup of TNBCs are inherently chemosensitive and achieve a pathological complete response (pCR) following combination anthracycline/taxane-based neo-adjuvant chemotherapy (NACT) [2,12-16]. These patients have a higher rate of survival compared to those with residual disease $[2,17,18]$. The basis for this variation in response to chemotherapy and survival remains unclear. TNBCs show considerable molecular heterogeneity, and no predictive markers have been identified to date [16, 17, 19-21]. Consequently, efforts have focused on modifying the standard chemotherapeutic regimen using new therapeutic agents to improve outcome.

The observation that many TNBCs exhibit dysfunction of DNA damage repair pathways $[22,23]$ has led to the inclusion of platinum salts in the treatment regimen with improved rates of pCR reported both in BRCA mutation carriers and in sporadic tumors in several phase II trials [12, 13, 24-29]. These data are encouraging; however, platinum salts are not yet part of the standard treatment for TNBC and a definite survival benefit remains to be demonstrated $[30,31]$. Despite this, carboplatin was added to the NACT regimen for TNBC in our institution in 2013 based on phase II trial data. The aim in this study was to examine the effect of carboplatin on outcome for patients with TNBC and to identify biomarkers predictive of response to treatment and outcome. Outcome data from a retrospective series of 333 patients diagnosed with TNBC, including patients treated with carboplatin added to anthracycline-taxane NACT, are presented. This cohort represents one of the largest clinical cohorts of TNBC in the literature, reported outside of the clinical trial setting.

\section{Materials and methods}

\section{Patient series}

The series comprised 333 consecutive patients who were diagnosed with TNBC at Galway University Hospital between January 1, 2000, and December 31, 2015. Cases were ER and PR negative by immunohistochemistry (IHC) [32] and HER2 negative by IHC and/or fluorescent in situ hybridization (FISH). Twenty-seven cases with low ER expression (Allred score 3) [32] were included. Tumor type and grade were re-evaluated $[33,34]$ on full-face sections of the resections or of the pre-treatment biopsy in cases where a pCR was achieved [GC, AS]. An adverse event was defined as a local ipsilateral recurrence; a contralateral breast cancer; a distant event [35], and each was recorded as TNBC or nonTNBC. A non-breast cancer in any organ outside the breast, excluding non-melanoma skin cancer, was recorded. Deaths from breast cancer were recorded, and non-cancer-related deaths were excluded.

Adjuvant chemotherapy was the standard-of-care for TNBC prior to 2010. This changed to NACT with an anthracycline-taxane combination after 2010 with carboplatin incorporated into the NACT regime from 2013. Four patients who had very low volume oligometastatic disease in distant sites shortly after diagnosis were treated with NACT and were included with this group for analysis based on the intention-to-treat. Sixty-eight patients did not receive chemotherapy for different reasons including metastatic disease diagnosed at $(n=13)$ or shortly after diagnosis $(n=2)$; patient declined or was unfit for treatment $(n=27)$ or for reasons that were not specified $(n=26)$. Patients who had metastasis at diagnosis were excluded from analyses of DFS and MFS.

Tumors were staged according to guidelines [36]. In accordance with recommendations for standardized reporting of pCR [15, 37], a pCR was defined as no residual invasive disease in the breast, with or without residual in situ disease, and no residual disease in the axillary lymph nodes (ypT0/isypN0) [36] and is referred to as pCR breast/axilla. For the purposes of statistical analysis, pCR breast (ypT0/ is) also was also examined as an endpoint.

\section{Immunohistochemistry}

TNBC status was confirmed by IHC using a tissue microarray (TMA) containing $2 \mathrm{~mm}$ tumor cores in cases that had 
sufficient tumor using ER (SP1 Rabbit Monoclonal antibody; Thermo-Scientific; Dilution 1:00); PR (16/SAN27 Mouse Monoclonal antibody; Lecia; Dilution 1:200); and HER2 (4B5 Rabbit Monoclonal antibody; Roche; pre-filled dilution) with HER2 FISH as required and reported according to current guidelines [38, 39]. Cases were classified as basal if there was any positivity for cytokeratin (CK) $5 / 6$ (D5/16, B4 Mouse Monoclonal antibody; Dako; Dilution 1:100) or epidermal growth factor receptor (EGFR) (EGFR.25; Leica; Dilution 1:100) [4] on full-face sections at diagnosis for some cases; on core biopsies for cases that had a pCR postNACT; and on TMA sections for other cases.

\section{Statistical analysis}

The Kruskal Wallis test with Dunn's post-estimation and Fisher's exact test were used to examine the differences in age and clinico-pathological characteristics between treatment groups, respectively. Differences in follow-up and survival times were analyzed using Oneway Anova with Bonferroni post-estimation. Associations between clinicopathological characteristics and survival were assessed using log-rank tests with two proportion tests to estimate the effect (difference in proportions) of significant variables. Pearson $\chi^{2}$ testing was used to assess the interaction between variables. Both univariate and multivariate Cox regression analyses were used to calculate hazard ratios (HR) and 95\% confidence intervals (95\% CI), adjusting for variables. Logistic regression analysis was used to calculate odds ratio (OR) and 95\% CI to adjust for prognostic variables. Test for trend of survivor testing assessed the impact of increasing prognostic variables on outcomes. Kaplan-Meier estimates were plotted for disease-free survival (DFS), metastases-free survival (MFS) and breast cancer-specific survival (BCSS). $p$ values reported were two-tailed, and $p<0.05$ was considered statistically significant. Statistical analysis was performed using Stata/IC (v14.0) and SPSS (v24).

\section{Results}

\section{Patient and tumor characteristics}

Patient demographics and tumor characteristic are shown in Table 1. Overall, $51 \%$ of patients received adjuvant chemotherapy $(n=168)$ and $29 \%(n=97)$ patients received NACT. Patients who received NACT were younger than those in the other treatment groups $(p<0.001)$. Tumor type differed between the groups $(p=0.004)$. Lobular carcinomas accounted for $5 \%$ of TNBCs, most of which were pleomorphic ILC, and were more common in those who did not receive chemotherapy. More of those who received NACT had grade 2 tumors compared to patients treated with adjuvant therapy $(p<0.001)$. The follow-up time was shorter for those who received NACT compared to those who received adjuvant therapy (median 30 months; range 5-126 and median 64 months, range 2-186, respectively), which paralleled the shift from adjuvant chemotherapy to NACT since 2010.

\section{Treatment}

NACT consisted of an anthracycline-taxane combination with platinum included for 31 patients. Paclitaxel was given weekly for 12 weeks with carboplatin administered at an $\mathrm{AUC}=5 \mathrm{mg} * \mathrm{~min} / \mathrm{mL}$ every 3 weeks, followed by dose dense doxorubicin plus cyclophosphamide. NACT was reasonably well tolerated, albeit with more toxicities recorded among those who received carboplatin: $19 \%(n=6)$ versus $13 \%$ $(n=8)$ had a treatment delay; $19 \%(n=6)$ versus $5 \%(n=3)$ failed to complete their recommended course of treatment; and 7\% $(n=2)$ versus $0 \%$ had a dose reduction in the platinum and non-platinum treated group, respectively. Despite failing to complete their recommended NACT, four of six patients treated with carboplatin achieved a pCR, whereas none of the three patients treated with non-platinum NACT who failed to complete their NACT achieved a pCR. In the adjuvant setting, most patients received a taxane; an anthracycline-taxane; or an anthracycline combination (Table 1). All adjuvant chemotherapy was well tolerated. Treatment delays were seen in 22\%, 20\% and 3\% among anthracycline, anthracycline-taxane and taxane combinations, respectively, and dose reductions were very infrequent $(<5 \%)$. Adjuvant endocrine therapy was given in the case of cancers with low $(<10 \%)$ ER or PR expression, or for an ER positive recurrence.

\section{Outcomes}

The median DFS, MFS and BCSS for all patients was 39.5, 40.5 and 44 months (range 0-199 months), respectively. The DFS, MFS and BCSS in the different treatment groups was compared (Fig. 1). There were statistically significant differences between the three treatment groups but there was no significant difference in DFS (log-rank test, $p=0.352$ ) or MFS (log-rank test, $p=0.094$ ) between patients receiving NACT versus adjuvant therapy. However, a marginally significant improvement in BCSS was seen in patients receiving adjuvant therapy compared to NACT (log-rank test, $p=0.049$ ).

The number of new adverse events in the different treatment groups was similar $(p=0.121)$ (Table 1$)$, and most of these $(67 \%, n=59)$ were distant recurrences of TNBC. Distant metastases were more common than local recurrences as a first event in the NACT group compared to the adjuvant therapy group $(p=0.029)$. Irrespective of 
Table 1 Patient and tumor characteristics

\begin{tabular}{|c|c|c|c|c|c|c|c|}
\hline \multicolumn{2}{|l|}{ Parameter } & \multirow{2}{*}{$\begin{array}{l}\text { All patients } \\
n(\%) \\
333\end{array}$} & \multirow{2}{*}{$\begin{array}{l}\text { NACT } \\
n(\%) \\
97(29 \%)\end{array}$} & \multirow{2}{*}{$\begin{array}{l}\text { Adjuvant CT } \\
n(\%) \\
168(51 \%)\end{array}$} & \multirow{2}{*}{$\begin{array}{l}\text { No CT } \\
n(\%) \\
68(20 \%)\end{array}$} & \multirow{2}{*}{$\begin{array}{l}p \text { Value } \\
\text { None versus } \\
\text { NACT versus } \\
\text { Adjuvant }\end{array}$} & \multirow{2}{*}{$\begin{array}{l}p \text { Value } \\
\text { NACT versus } \\
\text { Adjuvant CT }\end{array}$} \\
\hline Number of patients & & & & & & & \\
\hline \multirow[t]{2}{*}{ Age at diagnosis (years) } & Median & 55 & 48 & 55 & 75 & $<0.001$ & $<0.001$ \\
\hline & Range & 24-92 & $24-73$ & $30-79$ & 30-92 & & \\
\hline Menopausal & Pre & $119(36 \%)$ & $57(59 \%)$ & $58(35 \%)$ & $4(6 \%)$ & $<0.001$ & $<0.001$ \\
\hline \multirow[t]{2}{*}{ Status } & Post & $200(60 \%)$ & $33(34 \%)$ & $105(62 \%)$ & $62(91 \%)$ & & \\
\hline & Unknown & $14(4 \%)$ & $7(7 \%)$ & $5(3 \%)$ & $2(3 \%)$ & & \\
\hline \multirow[t]{4}{*}{ BRCA mutation } & None & $240(72 \%)$ & $69(71 \%)$ & $129(77 \%)$ & $42(62 \%)$ & 0.086 & 0.807 \\
\hline & BRCA1 mutation & $12(4 \%)$ & $4(4 \%)$ & $8(5 \%)$ & 0 & & \\
\hline & BRCA2 mutation & $7(2 \%)$ & $2(2 \%)$ & $5(3 \%)$ & 0 & & \\
\hline & Unknown & $74(22 \%)$ & $22(23 \%)$ & $26(15 \%)$ & $26(38 \%)$ & & \\
\hline \multirow{2}{*}{$\begin{array}{l}\text { Family history of breast } \\
\text { cancer }\end{array}$} & No & $123(55 \%)$ & $40(56 \%)$ & $66(54 \%)$ & $17(55 \%)$ & 0.956 & 0.763 \\
\hline & Yes & $101(45 \%)$ & $31(44 \%)$ & $56(46 \%)$ & $14(45 \%)$ & & \\
\hline \multirow[t]{7}{*}{ Tumor type } & Ductal & $271(81 \%)$ & $87(90 \%)$ & $138(82 \%)$ & $46(68 \%)$ & 0.004 & 0.595 \\
\hline & Lobular & $16(5 \%)$ & $1(1 \%)$ & $7(4 \%)$ & $8(15 \%)$ & & \\
\hline & Metaplastic & $15(5 \%)$ & $4(4 \%)$ & $6(4 \%)$ & $5(7 \%)$ & & \\
\hline & Medullary & $14(4 \%)$ & $4(4 \%)$ & $8(5 \%)$ & $2(3 \%)$ & & \\
\hline & Apocrine & $9(3 \%)$ & $1(1 \%)$ & $2(1 \%)$ & $6(9 \%)$ & & \\
\hline & Other $^{\mathrm{a}}$ & $4(1 \%)$ & 0 & $3(2 \%)$ & $1(1 \%)$ & & \\
\hline & Unknown & $4(1 \%)$ & 0 & $4(2 \%)$ & 0 & & \\
\hline \multirow[t]{4}{*}{ Tumor grade } & 1 & $1(0.5 \%)$ & 0 & $1(1 \%)$ & 0 & $<0.001$ & $<0.001$ \\
\hline & 2 & $63(19 \%)$ & $27(28 \%)$ & $15(9 \%)$ & $21(31 \%)$ & & \\
\hline & 3 & $265(79.5 \%)$ & $70(72 \%)$ & $148(88 \%)$ & $47(69 \%)$ & & \\
\hline & Unknown & $4(1 \%)$ & 0 & $4(2 \%)$ & 0 & & \\
\hline \multirow[t]{3}{*}{ Basal status $^{\mathrm{b}}$} & Positive & $254(76 \%)$ & $79(81 \%)$ & $131(78 \%)$ & $44(65 \%)$ & 0.225 & 0.227 \\
\hline & Negative & $57(17 \%)$ & $12(12 \%)$ & $31(18 \%)$ & $14(20 \%)$ & & \\
\hline & Unclassified & $22(7 \%)$ & $6(6 \%)$ & $6(4 \%)$ & $10(15 \%)$ & & \\
\hline \multirow[t]{5}{*}{ pT stage } & 1 & $78(33 \%)$ & NA & $57(34 \%)$ & $21(34 \%)$ & 0.088 & NA \\
\hline & 2 & $121(51 \%)$ & & $92(55 \%)$ & $29(48 \%)$ & & \\
\hline & 3 & $11(5 \%)$ & & $8(4.5 \%)$ & $3(5 \%)$ & & \\
\hline & 4 & $14(6 \%)$ & & $6(4 \%)$ & $8(13 \%)$ & & \\
\hline & Unknown & $12(5 \%)$ & & $5(3 \%)$ & 7 & & \\
\hline \multirow[t]{5}{*}{ pN stage } & 0 & $140(59 \%)$ & NA & $104(62 \%)$ & $36(53 \%)$ & 0.619 & NA \\
\hline & 1 & $43(18 \%)$ & & $33(20 \%)$ & $10(15 \%)$ & & \\
\hline & 2 & $19(8 \%)$ & & $12(7 \%)$ & $7(10 \%)$ & & \\
\hline & 3 & $14(6 \%)$ & & $8(5 \%)$ & $4(6 \%)$ & & \\
\hline & Unknown & $22(9 \%)$ & & $11(6 \%)$ & $11(16 \%)$ & & \\
\hline \multirow[t]{6}{*}{ ypT stage } & 0 & $35(36 \%)$ & $35(36 \%)$ & NA & NA & NA & NA \\
\hline & In situ & $8(8 \%)$ & $8(8 \%)$ & & & & \\
\hline & 1 & $27(28 \%)$ & $27(28 \%)$ & & & & \\
\hline & 2 & $12(13 \%)$ & $12(13 \%)$ & & & & \\
\hline & 3 & $5(5 \%)$ & $5(5 \%)$ & & & & \\
\hline & 4 & $10(10 \%)$ & $10(10 \%)$ & & & & \\
\hline \multirow[t]{5}{*}{ ypN stage } & 0 & $64(66 \%)$ & $64(66 \%)$ & NA & NA & NA & NA \\
\hline & 1 & $11(11 \%)$ & $11(11 \%)$ & & & & \\
\hline & 2 & $13(14 \%)$ & $13(14 \%)$ & & & & \\
\hline & 3 & $8(8 \%)$ & $8(8 \%)$ & & & & \\
\hline & Unknown & $1(1 \%)$ & $1(1 \%)$ & & & & \\
\hline
\end{tabular}


Table 1 (continued)

\begin{tabular}{|c|c|c|c|c|c|c|c|}
\hline Parameter & & $\begin{array}{l}\text { All patients } \\
n(\%)\end{array}$ & $\begin{array}{l}\text { NACT } \\
n(\%)\end{array}$ & $\begin{array}{l}\text { Adjuvant CT } \\
n(\%)\end{array}$ & $\begin{array}{l}\text { No CT } \\
n(\%)\end{array}$ & $\begin{array}{l}p \text { Value } \\
\text { None versus } \\
\text { NACT versus } \\
\text { Adjuvant }\end{array}$ & $\begin{array}{l}p \text { Value } \\
\text { NACT versus } \\
\text { Adjuvant CT }\end{array}$ \\
\hline \multirow[t]{2}{*}{ M stage } & 0 & $316(95 \%)$ & $93(96 \%)$ & $168(100 \%)$ & $55(81 \%)$ & \multirow[t]{2}{*}{$<0.001$} & \multirow[t]{2}{*}{0.008} \\
\hline & 1 & $17(5 \%)$ & $4(4 \%)$ & 0 & $13(19 \%)$ & & \\
\hline Chemotherapy & Anthra., Taxane & $124(47 \%)$ & $64(66 \%)$ & $60(36 \%)$ & NA & \multirow[t]{6}{*}{ NA } & \multirow[t]{6}{*}{ NA } \\
\hline \multirow[t]{5}{*}{ Agents $^{c}$} & Taxane & $64(24 \%)$ & 0 & $64(38 \%)$ & & & \\
\hline & $\begin{array}{l}\text { Anthra., Taxane, carbo- } \\
\text { platin }\end{array}$ & $31(12 \%)$ & $31(32 \%)$ & 0 & & & \\
\hline & Anthra & $30(11 \%)$ & 0 & $30(18 \%)$ & & & \\
\hline & Other & $6(2 \%)$ & 0 & $6(3 \%)$ & & & \\
\hline & Unknown regimen & $10(4 \%)$ & $2(2 \%)$ & $8(5 \%)$ & & & \\
\hline \multirow[t]{8}{*}{ Outcome } & All new adverse events ${ }^{\mathrm{d}, \mathrm{e}}$ & $88(28 \%)$ & $21(23 \%)$ & $46(27 \%)$ & $21(38 \%)$ & \multirow[t]{8}{*}{0.121} & \multirow[t]{8}{*}{0.395} \\
\hline & $\begin{array}{l}\text { Ipsilateral recurrence } \\
\text { (TNBC) }\end{array}$ & $13(4 \%)$ & $1(1 \%)$ & $8(5 \%)$ & $4(7 \%)$ & & \\
\hline & $\begin{array}{l}\text { Ipsilateral recurrence (non- } \\
\text { TNBC) }\end{array}$ & $2(0.6 \%)$ & 0 & $1(0.6 \%)$ & $1(2 \%)$ & & \\
\hline & Contralateral IBC (TNBC) & $3(1 \%)$ & 0 & $3(2 \%)$ & 0 & & \\
\hline & $\begin{array}{l}\text { Contralateral IBC (non- } \\
\text { TNBC) }\end{array}$ & $1(0.3 \%)$ & $1(1 \%)$ & 0 & 0 & & \\
\hline & Distant event & $59(19 \%)$ & $19(20 \%)$ & $26(15 \%)$ & $14(25 \%)$ & & \\
\hline & Other cancer (non-breast) & $10(3 \%)$ & 0 & $8(5 \%)$ & $2(4 \%)$ & & \\
\hline & Death from disease ${ }^{f}$ & $68(22 \%)$ & $18(19 \%)$ & $25(16 \%)$ & $25(39 \%)$ & & \\
\hline \multirow[t]{2}{*}{ Follow-up time (months) } & Median & 43 & 30 & 64 & 24 & \multirow[t]{2}{*}{$<0.001$} & \multirow[t]{2}{*}{$<0.001$} \\
\hline & Range & 0-199 & $5-126$ & $2-186$ & 0-199 & & \\
\hline
\end{tabular}

Anthra Anthracycline, $C T$ chemotherapy, $n$ number of patients, IBC invasive breast cancer, $N A C T$ neoadjuvant chemotherapy, $N A$ not applicable, $T N B C$ triple negative breast cancer

${ }^{a}$ Other tumor types include micropapillary $(n=1)$; mixed ductal-lobular $(n=1)$, mixed ductal-micropapillary $(n=1)$ and papillary carcinoma $(n=1)$; ILC comprised pleomorphic ILC $(n=13)$ and classic ILC $(n=3)$

${ }^{\mathrm{b}}$ Basal status: any positivity for either cytokeratin $5 / 6$ or EGFR by immunohistochemistry

${ }^{\mathrm{c}}$ Chemotherapeutic agents listed for patients known to have received either adjuvant chemotherapy or NACT

${ }^{\mathrm{d}}$ All new adverse events were classified according to Maastricht Delphi consensus criteria [35]. All distant events of breast cancer were TNBC. There were no adverse events that were either regional axillary or in situ disease. Non-melanoma skin cancer was excluded from other cancer that was non-breast. Metastatic disease at diagnosis was not regarded as a new event

${ }^{\mathrm{e}}$ Number of patients evaluable for new adverse events excluding death from disease (DFS, MFS) =316: NACT $(n=93)$, adjuvant chemotherapy $(n=168)$, no CT $(n=55)$

${ }_{\mathrm{f}}^{\mathrm{f}}$ Number of patients evaluable for death $(\mathrm{BCSS})=321$ : NACT $(n=96)$, adjuvant therapy $(n=161)$, no chemotherapy $(n=64)$

whether local recurrence or distant metastatic disease developed first, there was no difference in the number of patients who ultimately developed metastatic disease in the NACT or adjuvant therapy groups $(p=0.527)$. A new non-TNBC breast cancer was uncommon $(3 \%, n=3)$; as was a cancer outside the breast $(11 \%, n=10)$, most of which occurred in those who received adjuvant therapy, possibly due to their longer follow-up time. Overall, 22\% $(n=68)$ of patients died from TNBC; this included 64 patients who had non-metastatic disease at diagnosis. The time from recurrence to death was short for all patients (median 8 months; 0-33). There were no treatment-related deaths.

\section{Response to NACT}

A pCR breast (ypT0/is) was observed in 43 patients (44\%), including eight who had residual in situ disease only; 37 patients (38\%) had a pCR breast/axilla (ypT0/isN0). A pCR breast was significantly more common in patients who received carboplatin $(n=18,58 \%)$ compared to those who did not $(n=23,36 \%)$ (Pearson $\left.\chi^{2}=4.81 ; p=0.041\right)$. Likewise, a pCR breast/axilla was more frequent with carboplatin therapy $(n=17,55 \%)$ than without it $(n=18,28 \%)$ (Pearson $\left.\chi^{2}: 6.41, p=0.011\right)$. Univariate logistic regression analysis (Table 2) revealed that the likelihood of both a pCR breast and pCR breast/axilla was highest after carboplatin 

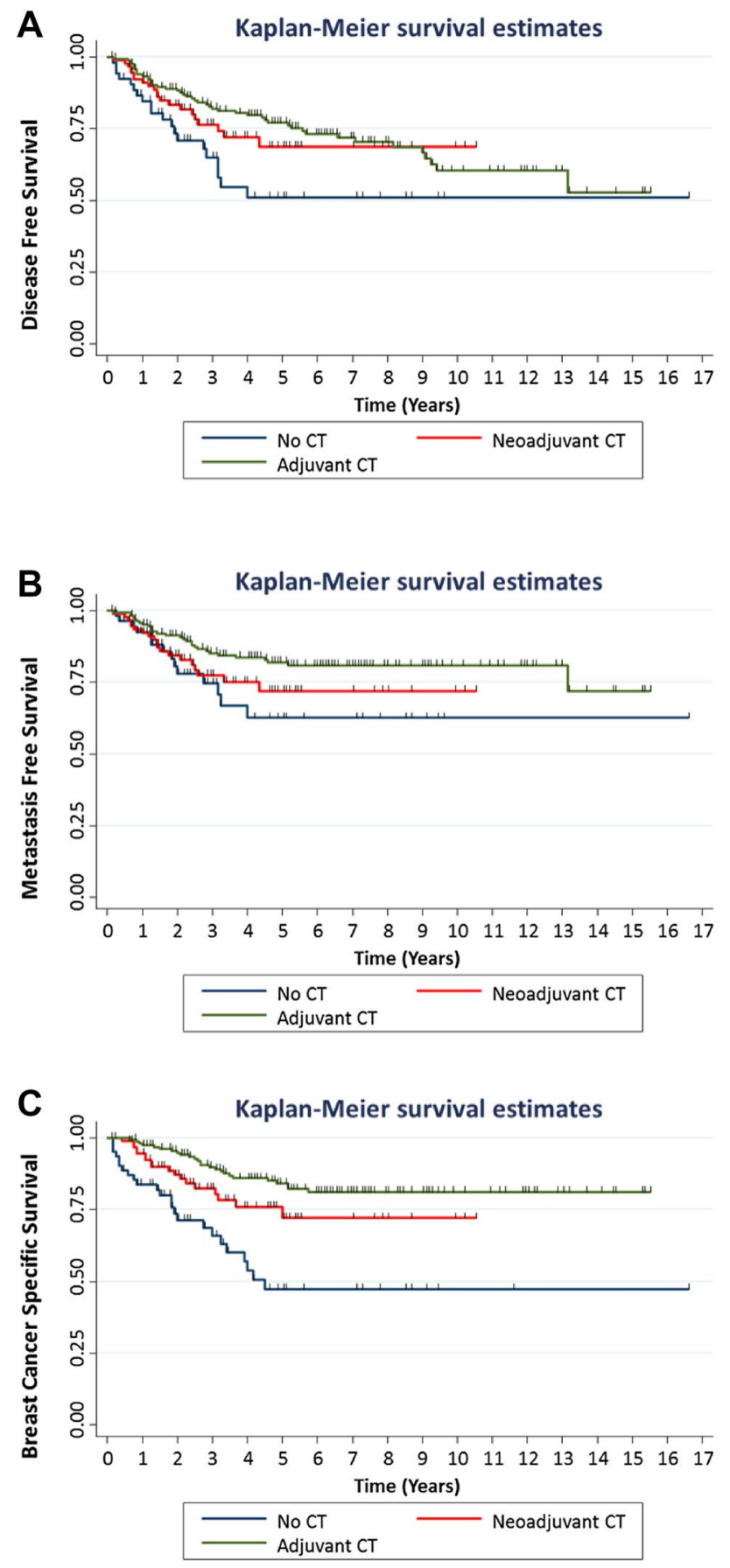

Fig. 1 Disease-free, metastases-free and breast cancer-specific survival for patients according to schedule of chemotherapy. KaplanMeier cumulative survival curves for $\mathbf{a}$ disease-free survival and $\mathbf{b}$ metastases-free survival for patients who received adjuvant chemotherapy (CT) $(n=168)$, neoadjuvant CT $(n=93)$ or no CT $(n=55)$ (log-rank test DFS, $p$ value $=0.024$ ) and (log-rank test MFS, $p$ value $=0.045$ ). $\mathbf{c}$ Kaplan-Meier cumulative survival curves for breast cancer-specific survival for patients for patients who received adjuvant therapy $(n=161)$, neoadjuvant CT $(n=96)$ or no CT $(n=64)$ (log-rank test, $p$ value $<0.001$ )
Table 2 Univariate and multivariable analyses of predictors of pCR breast and pCR breast/axilla

\begin{tabular}{|c|c|c|c|c|}
\hline & $N$ & $\mathrm{OR}^{\mathrm{a}}$ & $95 \% \mathrm{CI}$ & $p$ Value \\
\hline \multicolumn{5}{|l|}{ Univariate analysis } \\
\hline \multicolumn{5}{|l|}{ pCR breast } \\
\hline Age at diagnosis & 97 & 1.02 & $0.98-1.05$ & 0.434 \\
\hline Menopausal status & 90 & 1.06 & $0.45-2.52$ & 0.895 \\
\hline Family history of breast cancer & 71 & 0.34 & $0.13-0.90$ & 0.030 \\
\hline Tumor type & 97 & 0.91 & $0.65-1.26$ & 0.557 \\
\hline Tumor grade & 97 & 0.15 & $0.05-0.47$ & 0.001 \\
\hline Basal status ${ }^{\mathrm{b}}$ & 91 & 0.63 & $0.18-2.26$ & 0.477 \\
\hline Platinum-based NACT & 95 & 0.38 & $0.16-0.91$ & 0.030 \\
\hline \multicolumn{5}{|l|}{ pCR breast/axilla } \\
\hline Age at diagnosis & 97 & 1.01 & $0.98-1.05$ & 0.454 \\
\hline Menopausal status & 90 & 1.1 & $0.45-2.67$ & 0.833 \\
\hline Family history of breast cancer & 71 & 0.51 & $0.19-1.32$ & 0.162 \\
\hline Tumor grade & 97 & 0.08 & $0.02-0.36$ & 0.001 \\
\hline Tumor type & 97 & 1.04 & $0.73-1.48$ & 0.815 \\
\hline Basal status ${ }^{\mathrm{b}}$ & 91 & 0.49 & $0.12-1.95$ & 0.311 \\
\hline Platinum-based NACT & 95 & 0.32 & $0.13-0.79$ & 0.013 \\
\hline \multicolumn{5}{|l|}{ Multivariable analysis } \\
\hline pCR breast & 95 & & & \\
\hline Age at diagnosis & & 1.00 & $0.96-1.05$ & 0.892 \\
\hline Tumor grade & & 0.10 & $0.03-0.37$ & 0.001 \\
\hline Platinum-based NACT & & 0.26 & $0.09-0.74$ & 0.011 \\
\hline pCR breast/axilla & 95 & & & \\
\hline Age at diagnosis & & 1.01 & $0.97-1.05$ & 0.704 \\
\hline Tumor grade & & 0.05 & $0.01-0.27$ & $<0.001$ \\
\hline Platinum-based NACT & & 0.17 & $0.06-0.54$ & 0.002 \\
\hline
\end{tabular}

$n$ Number of patients, $O R$ odds ratio, $C I$ confidence interval, NACT neoadjuvant chemotherapy, $p C R$ pathological complete response

${ }^{a} \mathrm{OR}$ for a non-pCR by Logistic regression analysis

${ }^{\mathrm{b}}$ Basal status: any positivity for either cytokeratin $5 / 6$ or EGFR by immunohistochemistry

$\left(\mathrm{OR}_{\text {non-pCR }}=0.38 ; 95 \%\right.$ CI $0.16-0.91, p=0.030 ;$ OR 0.32; $95 \%$ CI $0.13-0.79 ; p=0.013$, respectively); and in grade 3 tumors (OR 0.15; 95\% CI 0.05-0.47; $p=0.001$; OR 0.08; $95 \%$ CI $0.02-0.36 ; p=0.001$, respectively). A family history of breast cancer increased the likelihood of a pCR breast (OR 0.34; 95\% CI 0.13-0.90; $p=0.030$ ) but not of a pCR breast/axilla (OR $0.51 ; 95 \%$ CI $0.19-1.31 ; p=0.162) ; 61 \%$ of those with a family history of breast cancer achieved a pCR breast compared to $35 \%$ of those without a family history (Pearson $\left.\chi^{2}=4.85 ; p=0.028\right)$. BRCA1/2 mutation status did not increase the likelihood of a $\mathrm{pCR}$, but four of the six BRCA1/2 mutation carriers had a pCR breast, and the remaining two patients had a near total response (ypT1mi or ypT1b). None of the other parameters were associated with pCR on univariate analysis.

By multivariable analysis (Table 2), carboplatin therapy (OR 0.26 ; 95\% CI $0.09-0.74 ; p=0.011)$ and grade 3 
histology (OR 0.10; 95\% CI 0.03-0.37; $p=0.001$ ) remained independently associated with a pCR breast and with a pCR breast/axilla (OR $0.17 ; 95 \%$ CI $0.06-0.54 ; p=0.002$, and OR $0.05 ; 95 \%$ CI $0.01-0.27 ; p<0.001$ for carboplatin and grade, respectively).

\section{DFS, MFS and BCSS}

\section{Patients treated with NACT}

By univariate analyses (Supplementary Tables 1 and 2), both pCR endpoints increased the likelihood of an improved DFS, MFS and BCSS. The magnitude of the effect of pCR on outcome was greatest for pCR breast/axilla for DFS $(\mathrm{HR}=6.66$; 95\% CI $1.54-28.58 ; p=0.011)$; for MFS (HR $=5.90 ; 95 \%$ CI 1.36-25.56; $p=0.018)$; and for BCSS (HR $=10.30 ; 95 \%$ CI 1.37-77.38; $p=0.023$ ) (Fig. 2).

There was a significant trend of association between increasing ypT category and adverse DFS $\left(\chi^{2}\right.$ test for trend, 13.56; $p=0.002)$, MFS ( $\chi^{2}$ test for trend, $12.90 ; p=0.0003$ ) and BCSS $\left(\chi^{2}\right.$ test for trend, 11.14; $\left.p=0.0008\right)$. However, there was no significant difference in outcome for patients with ypT1 versus ypT0/ypT0/is disease. A significant trend of association was also observed between an increasing number of positive nodes and adverse DFS ( $\chi^{2}$ test for trend, $18.32 ; p<0.0001)$, MFS ( $\chi^{2}$ test for trend, $17.20 ; p<0.001$ ) and BCSS ( $\chi^{2}$ test for trend, 33.56; $p<0.001$ ). The outcome was worst for those with ypN3 disease (Supplementary Tables 1 and 2).

On multivariable analyses (Table 3), a pCR breast/axilla was the only independent predictor of DFS (HR $=6.23$; 95\% CI $1.36-28.50 ; p=0.018)$, MFS (HR $=5.08 ; 95 \%$ CI $1.09-23.65 ; p=0.038)$ and BCSS (HR $=8.52 ; 95 \%$ CI $1.09-66.64 ; p=0.041$ ), when adjusted for age at diagnosis, tumor grade, tumor type, basal status and the administration of carboplatin. In a separate multivariable model using the same co-variables, pCR breast also remained the only independent predictor of DFS, MFS and BCSS (Supplementary Table 3).

The association between carboplatin therapy and outcome limited to 24-month follow-up time was examined (Fig. 3) because the median follow-up time for patients who received carboplatin was short (18 months; range 8-34). DFS was improved with platinum therapy but the association did not reach statistical significance on univariate $\left(\mathrm{HR}_{\text {non-pCR }}=0.34\right.$; 95\% CI 0.75-1.50; $p=0.153$ ) or multivariable analysis (Supplementary Table 4$)$.

\section{Patients who did not receive NACT}

Univariate analysis (Supplementary Tables 1 and 2) revealed that increasing tumor size (pT) and nodal status were a significant risk factor for adverse DFS $\left(\chi^{2}\right.$ test trend 8.83 ,
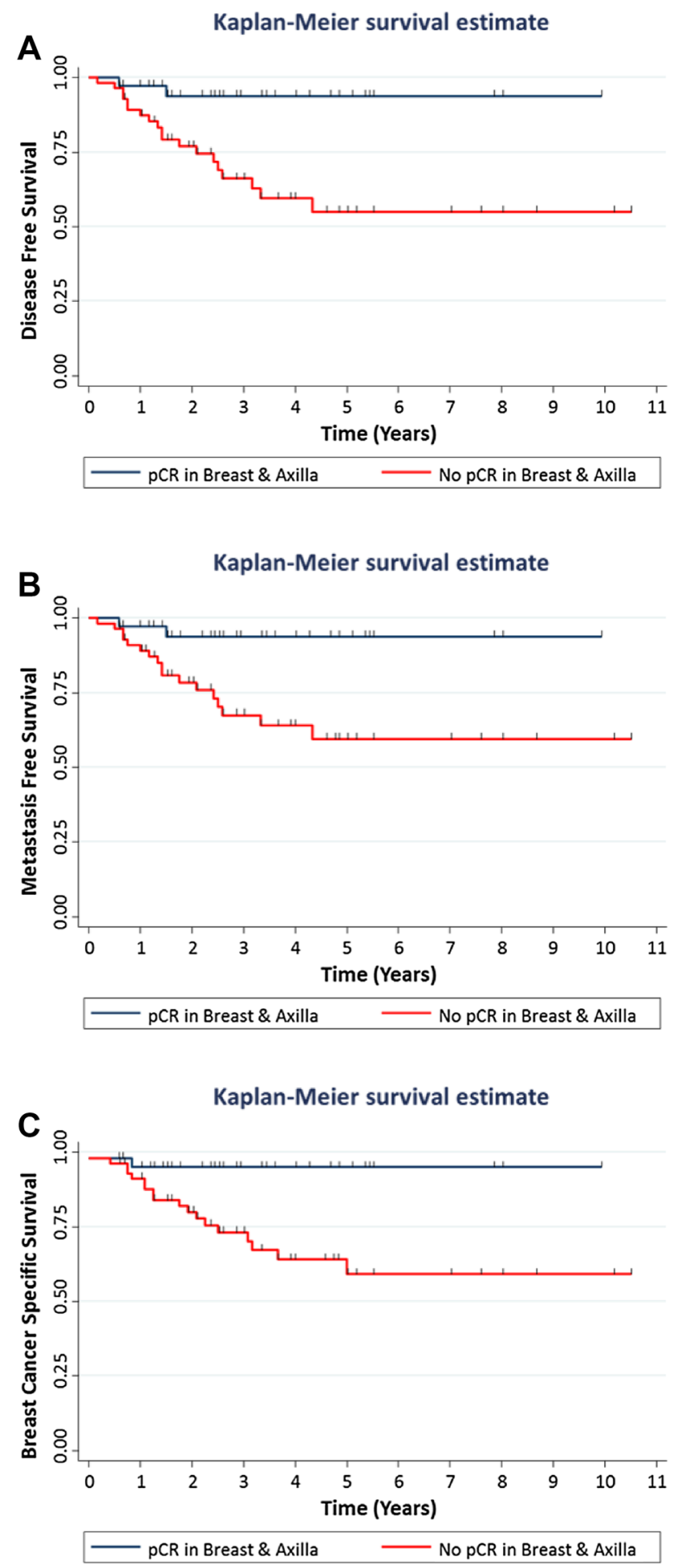

Fig. 2 Disease-free, metastases-free and breast cancer-specific survival according to pathological response to NACT. Kaplan-Meier cumulative survival curves show the association between pathological complete response (pCR) breast/axilla and a disease-free survival $(n=93)$ log-rank test, $p$ value $=0.003 ; \mathbf{b}$ metastases-free $(n=93)$ log-rank test, $p$ value $=0.007$; and $\mathbf{c}$ breast cancer-specific survival $(n=96)$ log-rank test, $p$ value $=0.005$. A pCR breast/axilla is defined as ypT0/isN0 
Table 3 Multivariable analysis of DFS, MFS and BCSS for patients treated with NACT

\begin{tabular}{|c|c|c|c|c|}
\hline & $n$ & $\mathrm{HR}^{\mathrm{a}}$ & $95 \% \mathrm{CI}$ & $p$ Value \\
\hline Disease-free survival & 85 & & & \\
\hline Age at diagnosis & & 0.99 & $0.95-1.04$ & 0.776 \\
\hline Tumor grade & & 1.05 & $0.39-2.81$ & 0.926 \\
\hline Tumor type & & 0.64 & $0.27-1.49$ & 0.298 \\
\hline Basal status ${ }^{\mathrm{b}}$ & & 1.61 & $0.36-7.20$ & 0.530 \\
\hline Platinum-based therapy & & 0.68 & $0.18-2.56$ & 0.566 \\
\hline pCR breast/axilla ${ }^{\mathrm{c}}$ & & 6.23 & $1.36-28.50$ & 0.018 \\
\hline Metastasis-free survival & 85 & & & \\
\hline Age at diagnosis & & 0.99 & $0.95-1.04$ & 0.841 \\
\hline Tumor grade & & 0.85 & $0.31-2.37$ & 0.759 \\
\hline Tumor type & & 0.67 & $0.30-1.52$ & 0.343 \\
\hline Basal status ${ }^{\mathrm{b}}$ & & 1.47 & $0.32-6.63$ & 0.619 \\
\hline Platinum-based therapy & & 0.69 & $0.18-2.65$ & 0.590 \\
\hline pCR breast/axilla ${ }^{\mathrm{c}}$ & & 5.08 & $1.09-23.65$ & 0.038 \\
\hline Breast cancer-specific survival & 88 & & & \\
\hline Age at diagnosis & & 1.01 & $0.96-1.06$ & 0.714 \\
\hline Tumor grade & & 1.14 & $0.40-3.26$ & 0.813 \\
\hline Tumor type & & 0.77 & $0.40-1.49$ & 0.441 \\
\hline Basal status ${ }^{\mathrm{b}}$ & & 1.32 & $0.29-6.07$ & 0.719 \\
\hline Platinum-based therapy & & 0.310 & $0.04-2.50$ & 0.272 \\
\hline pCR breast/axilla ${ }^{\mathrm{c}}$ & & 8.52 & $1.09-66.64$ & 0.041 \\
\hline
\end{tabular}

$n$ Number of patients, $H R$ hazard ratio, $C I$ confidence interval

${ }^{\mathrm{a} C o x}$ regression survival analysis

${ }^{b}$ Basal status: any positivity for either cytokeratin $5 / 6$ or EGFR by immunohistochemistry

${ }^{\mathrm{c}} \mathrm{HR}$ for non-pCR using pCR as the baseline value

$p=0.003$; and 29.06, $p<0.001$, respectively); MFS $\left(\chi^{2}\right.$ test trend 24.92, $p<0.001)$ and BCSS $\left(\chi^{2}\right.$ test trend 32.40, $p<0.001$ and 29.46, $p<0.001$, respectively). A strong positive correlation between tumor size and nodal status (Pearson $\chi^{2}=73.01, p<0.001$ ) was noted. However, there was no difference in outcome for $\mathrm{pT} 2$ and pT1 tumors or between $\mathrm{pN} 1$ and node negative disease.

Multivariable analysis (Table 4) revealed that only nodal stage at diagnosis was an independent predictor of DFS $(\mathrm{HR}=1.75 ; 95 \%$ CI $1.30-2.34 ; p<0.001)$, and both nodal status $(\mathrm{HR}=1.58 ; 95 \% \mathrm{CI} 1.13-2.23 ; p=0.008)$ and tumor size $(\mathrm{HR}=1.68$; 95\% CI $1.10-2.58 ; p=0.018)$ were independent predictors of MFS. When those with M1 at diagnosis were excluded, $\mathrm{pT}$ was the only independent predictor of BCSS $(\mathrm{HR}=2.08 ; 95 \%$ CI $1.39-3.11 ; p<0.001)$. In a separate analysis including those with M1 disease at diagnosis, metastasis and tumor size were independent predictors of BCSS.

There was no association between tumor type and outcome (Table 3, Supplementary Tables 1,2). Metaplastic carcinomas were uncommon $(n=15)$; however, we observed

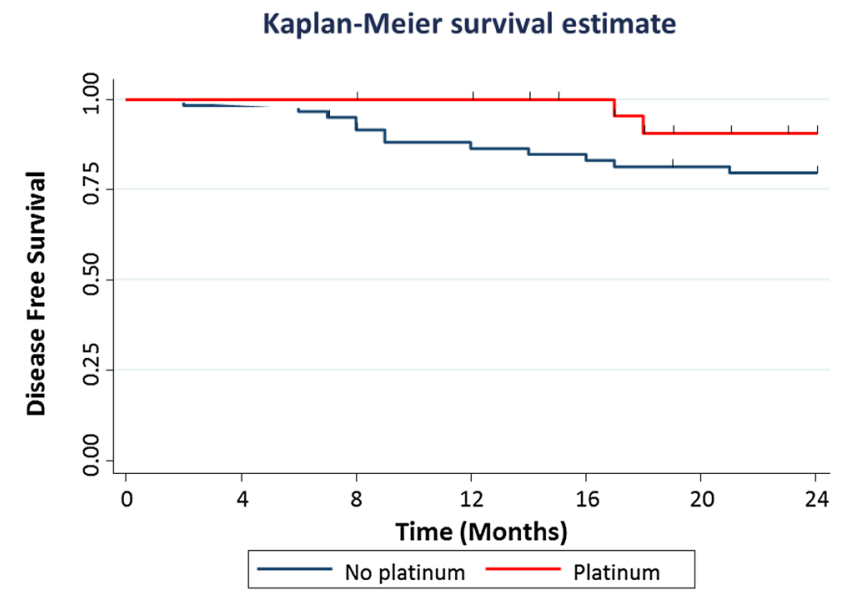

Fig. 3 Disease-free survival for patients who received NACT according to the administration of platinum agents. Kaplan-Meier curves show the disease-free survival for patients stratified according to the administration of platinum-based NACT. The analysis was confined to 24-month follow-up period because the follow-up time for patients who received a platinum agent was short. A new event was observed in two of 31 patients who received a platinum and in 12 of 60 patients who received standard anthracycline-taxane-based NACT (log-rank test, $p$ value $=0.262$ )

Table 4 Multivariable analysis of DFS, MFS and BCSS in patients treated with adjuvant or no chemotherapy

\begin{tabular}{lcccr}
\hline & $n$ & HR $^{\mathrm{a}}$ & $95 \%$ CI & $p$ Value \\
\hline Disease-free survival & 206 & & & \\
Age at diagnosis & & 1.02 & $0.99-1.04$ & 0.128 \\
Tumor grade & & 0.78 & $0.40-1.51$ & 0.455 \\
pT & & 1.07 & $0.73-1.59$ & 0.723 \\
pN & & 1.75 & $1.30-2.34$ & $<0.001$ \\
Metastases-free survival & 206 & & & \\
Age at diagnosis & & 1.01 & $0.99-1.04$ & 0.275 \\
Tumor grade & & 1.18 & $0.47-3.04$ & 0.340 \\
pT & & 1.70 & $1.10-2.62$ & 0.017 \\
pN & 1.57 & $1.11-2.21$ & 0.009 \\
Breast cancer-specific survival & 203 & & & \\
Age at diagnosis & & 1.01 & $0.99-1.04$ & 0.238 \\
Tumor grade & 1.46 & $0.57-3.76$ & 0.427 \\
pT & 2.05 & $1.37-3.10$ & 0.001 \\
pN & 1.30 & $0.91-1.84$ & 0.154 \\
M1 at diagnosis & 7.66 & $2.72-20.08$ & $<0.001$ \\
\hline
\end{tabular}

$n$ number of patients, $H R$ hazard ratio, $C I$ confidence interval, $n c$ not calculable, $M 1$ metastatic disease

${ }^{\mathrm{a}} \mathrm{HR}$ for non-pCR by Cox regression survival analysis using $\mathrm{pCR}$ as the baseline value

that only five of the 15 patients died from disease after a median time to recurrence of 26 months (range $0-44$ months) and median time to death of 32 months (range 2-58 months). There was no difference in survival for patients 
with metaplastic carcinoma between the three treatment groups.

\section{Discussion}

We show in a retrospective series that patients with TNBC who received carboplatin with anthracycline-taxane-based NACT have significantly higher rate of pCR compared to those who received anthracycline/taxane alone. A pCR breast and a pCR breast/axilla, achieved in 58\% and 55\% of those treated with carboplatin, respectively, were the only independent predictors of survival, although follow-up time was short. In patients who did not received NACT, only traditional parameters of nodal status and tumor size had prognostic significance.

Several studies have shown the favorable association between pCR and outcome [40-43]; patients with TNBC who attain a pCR have an outcome that is comparable to that for patients with non-TNBC who attain a pCR [2, 14, $15,44]$. It is generally accepted that a pCR breast/axilla is a stronger predictor of outcome than a pCR breast, which was shown in our series where a pCR breast/axilla was a stronger predictor of BCSS than a pCR breast for analyses of BCSS. However, this difference in benefit was not observed for DFS or MFS on multivariate analysis, where both pCR endpoints improved DFS and MFS comparably. The benefit of a pCR in terms of survival shown in our data has been reported by others $[13,15,45,46]$. However, the prognostic value of pCR has not yet been validated at trial level, as reported in the pooled analysis of almost 12,000 patients [15]; and it is likely that other variables affect the relationship between pCR and survival that should be examined in future trials examining the prognostic value of pCR [46, 47].

The rate of pCR in our series is comparable to that reported in two prospective randomized phase II clinical trials evaluating carboplatin with anthracycline-taxane NACT in stage II-III TNBC. In the GeparSixto trial, carboplatin and bevacizumab resulted in an increase from 37 to $53 \%$, in the rate of $\mathrm{pCR}$ in 158 patients [13]. The Alliance study [12] reported a pCR of 54\% in those treated with carboplatin with an alkylating agent. Similarly, in a smaller series, Ando et al. [26] reported a pCR in $61 \%$ for those treated with carboplatin with anthracycline-taxane. Only the earlier GEICAM randomized trial failed to show any improvement in pCR rate with carboplatin [48]. The phase II adaptively randomized I-SPY 2 study [25] which assessed the addition of a poly-ADP-ribose-polymerase (PARP) inhibitor, veliparib, plus carboplatin to anthracycline-taxane NACT also reported a pCR in $51 \%$ of those who received veliparib and carboplatin.

The data from these studies are promising; however, the optimal way of incorporating platinum agents in the NACT regimen is not yet established [49]. In the GeparSixto and Ca.Pa.Be studies, different anthracycline-taxane combinations were used and bevacizumab was given $[13,28]$. The dose of carboplatin also differed between studies: GEICAM [48], CALGB 40603 [12] and I-SPY2 [25] used carboplatin every 3 weeks at an $\mathrm{AUC}=6$; Ando et al. [26] used carboplatin every 3 weeks at an $\mathrm{AUC}=5$; Ca.Pa.Be [28] used weekly carboplatin at $\mathrm{AUC}=2$ and the GeparSixto (13) used weekly carboplatin at an $\mathrm{AUC}=1.5$. In our institution, paclitaxel is given weekly for 12 weeks with carboplatin administered at an AUC $=5$ every 3 weeks, followed by dose dense doxorubicin plus cyclophosphamide. Platinum agents are reportedly associated with increased toxicity relative to the standard chemotherapeutic regimen $[12,13]$. Some suggest that carboplatin plus paclitaxel may have less hematological toxicities than carboplatin plus docetaxel [50-52] but others report good tolerance of carboplatin plus docetaxel in an anthracycline-free regimen [53]. In our cohort, toxicities and treatment delays were increased in those who received carboplatin, nonetheless most of those who received carboplatin but did not complete their still achieved a pCR.

Tumor grade was the only other independent predictor of pCR in this study and is in keeping with reported increased chemo-sensitivity for grade 3 compared to lowgrade TNBCs [16]. This emphasizes the importance of accurate tumor grading in pre-NACT tumor material. Although most TNBCs are grade 3, we and others have recorded that between 16 and $21 \%$ of TNBCs are not high grade in series that exclude the low-grade indolent subtypes of TNBC [54, 55 , present series]. Tumour histological type was not a prognostic factor in our series. The proportion of ILC in our series $(5 \%)$ was greater than the $1 \%$ reported by others in 20,000 to 90,000 TNBCs [54-56]. However, it was comparable to the $7.7 \%$ of ILC recorded in a series 841 TNBCs [57], which suggests that the discrepancy may relate to the size of series. Metaplastic carcinomas were also uncommon, but we noted that two-thirds of patients with this subtype were disease-free after a median follow-up of 40 months (range 2-154 months). None of the other parameters examined in this study had prognostic or predictive significance.

Basal status by IHC was not informative, which contrasts with reports of adverse outcome for basal TNBCs [4] and with data showing higher rates of pCR for 'Basal-like 1' TNBCs defined by the TNBC type- $4[16,58]$. There is, as yet, no consensus on either the definition or on the clinical significance of the basal phenotype. Tumors identified as basal using different platforms overlap $[4,6,16,17,19,20$, 59], but different platforms do not characterize the same tumors as basal. The majority of TNBCs in our series arose in the sporadic setting; however, a pCR breast was significantly associated with a family history of breast cancer, which may point to enhanced chemo-sensitivity or possibly 'BRCA-ness' in those with a family history [22, 23]. 
The overall outcome and pattern of recurrence for all patients in our series is in line with that reported by others [1, 2, 4-8]. Early distant metastases were more common that local recurrences as a first event, and the median survival time from first was only 8 months. In the non-neoadjuvant setting, tumor size and nodal status were the only predictors of outcome with a significant positive trend of association between these variables and outcome observed. However, there was no survival difference between patients with pT2 versus pT1 disease or between those with $\mathrm{pN} 1$ versus $\mathrm{pN} 0$ disease. This is at variance with other reports [5], in which outcome was related to nodal positivity versus node negative disease and not the number of positive nodes. This inconsistency may be explained by the small number of cases with N1 disease $(n=48)$ in our series. A nonlinear correlation between both nodal status and tumor size with outcome in TNBC has been observed by others $[1,60,61]$ which suggests that tumor size and extent may be less valid for TNBC with its more aggressive biological behavior than for breast cancer as a whole.

There were limitations with this study. First, the median follow-up time for the NACT group was 30 months and only 18 months for those received carboplatin, reflecting the change in chemotherapy practice over time. Notwithstanding, the natural history of early recurrences peaking between within 3 years of diagnosis for TNBCs [1, 2, 4-8] mitigates to some degree the effect of short follow-up time on DFS. Also, the size of our series was not large enough to explore prognostic differences between subsets of TNBC. However, this study represents one of the largest clinical TNBC cohorts reported; we identified very few studies, performed outside of clinical trials, with larger cohorts of TNBC patients [5, 62-64]. These focused on surgical procedures [62, 63] or loco-regional recurrences as primary outcomes [64-66]. Even when clinical trials are included, this cohort represents a large, significant patient sample as only the BEATRICE $(n=2591)$ [65], GeparQuinto $(n=663)$ [66], NSABP-B40 $(n=479)$ [67] and CALGB $40603(n=433)$ [12] trials had larger TNBC patient cohorts.

The ypT stage assigned by the reporting breast pathologist at the time of diagnosis was used for analyses. Other systems that measure the degree of response to chemotherapy [68, 69] were reported inconsistently before 2013 and were not used for analysis. The Residual Cancer Burden score may be more promising than the TNM system for post-NACT staging; it provides an index that is predictive of long-term survival with reports of good reproducibility [37, 69-71]. Finally, 27 patients with low ER expression (1-9\% positivity) were included. The optimal treatment for these 'ER-poor' cancers is not defined. In our institution, these are treated as ER negative disease, although some receive adjuvant endocrine therapy. Whether including these patients could skew our results is a legitimate question; however, molecular subtype analysis suggests that the majority of these cancers have the same profiles and clinical outcome as ER negative disease [72, 73].

In summary, our data demonstrate that carboplatin added to anthracycline-taxane NACT significantly improves the rate of pCR in TNBC and that pCR is the only independent predictor of outcome, albeit at relatively short followup time. Grade 3 histology was the only other independent predictor of $\mathrm{pCR}$, underscoring the importance of accurate evaluation of grade in TNBCs pre-NACT. Platinum therapy is not yet standard-of-care for TNBC. While awaiting results from phase III trials examining the survival advantage for platinums (NCT02488967; NCT02445391), our results add to the data supporting the incorporation of carboplatin with anthracycline-taxane NACT [74]. It remains unclear whether all or a subset of TNBCs derive benefit from platinum and identification of predictive biomarkers of response are required.

Acknowledgements The authors are thankful to Dr Helen Ingoldsby for providing pathology support and acknowledge the support of Ms Terri Muldoon and Mr John Folan of the Department of Anatomic Pathology at GUH for retrieving pathology material.

Funding Breast Cancer Now (2013MayPR019 and 2015NovPhD643), Science Foundation Ireland (17/CDA/4638) and Irish Cancer Society (SG); Breast Cancer Research (EW, MJK), NUI Galway School of Medicine Scholarship (EW).

Data availability Data that support this study are available upon reasonable request.

\section{Compliance with ethical standards}

Conflict of interest The authors declare that they have no conflicts of interest.

Ethical standards This work complies with regulations governing ethical standards. Informed consent was obtained from patients who participated in this study, and the project was approved by the Clinical Research Ethics Committee, GUH (Ref. CA1012) on January 23, 2014.

Open Access This article is distributed under the terms of the Creative Commons Attribution 4.0 International License (http://creativeco mmons.org/licenses/by/4.0/), which permits unrestricted use, distribution, and reproduction in any medium, provided you give appropriate credit to the original author(s) and the source, provide a link to the Creative Commons license, and indicate if changes were made.

\section{References}

1. Dent R, Trudeau M, Pritchard KI, Hanna WM, Kahn HK, Sawka CA et al (2007) Triple-negative breast cancer: clinical features and patterns of recurrence. Clin Cancer Res 13(15):4429-4434

2. Liedtke C, Mazouni C, Hess KR, André F, Tordai A, Mejia JA et al (2008) Response to neoadjuvant therapy and long-term 
survival in patients with triple-negative breast cancer. J Clin Oncol 26(8):1275-1281

3. Foulkes WD, Smith IE, Reis-Filho JS (2010) Triple-negative breast cancer. N Engl J Med 363(20):1938-1948

4. Cheang MCU, Voduc D, Bajdik C, Leung S, McKinney S, Chia SK et al (2008) Basal-like breast cancer defined by five biomarkers has superior prognostic value than triple-negative phenotype. Clin Cancer Res 14(5):1368-1376

5. Hernandez-Aya LF, Chavez-MacGregor M, Lei X, Meric-Bernstam F, Buchholz TA, Hsu L et al (2011) Nodal status and clinical outcomes in a large cohort of patients with triple-negative breast cancer. J Clin Oncol 29(19):2628-2634

6. Sørlie T, Perou CM, Tibshirani R, Aas T, Geisler S, Johnsen H et al (2001) Gene expression patterns of breast carcinomas distinguish tumor subclasses with clinical implications. Proc Natl Acad Sci USA 98(19):10869-10874

7. Kennecke H, Yerushalmi R, Woods R, Cheang MCU, Voduc D, Speers CH et al (2010) Metastatic behavior of breast cancer subtypes. J Clin Oncol 28(20):3271-3277

8. Thike AA, Iqbal J, Cheok PY, Chong APY, Tse GM-K, Tan B et al (2010) Triple negative breast cancer: outcome correlation with immunohistochemical detection of basal markers. Am J Surg Pathol 34(7):956-964

9. Dent R, Hanna WM, Trudeau M, Rawlinson E, Sun P, Narod SA (2009) Pattern of metastatic spread in triple-negative breast cancer. Breast Cancer Res Treat 115(2):423-428

10. Abramson VG, Mayer IA (2014) Molecular heterogeneity of triple-negative breast cancer. Curr Breast Cancer Rep 6(3):154-158

11. Badve S, Dabbs DJ, Schnitt SJ, Baehner FL, Decker T, Eusebi V et al (2011) Basal-like and triple-negative breast cancers: a critical review with an emphasis on the implications for pathologists and oncologists. Mod Pathol 24(2):157-167

12. Sikov WM, Berry DA, Perou CM, Singh B, Cirrincione CT, Tolaney SM et al (2015) Impact of the addition of carboplatin and/ or bevacizumab to neoadjuvant once-per-week paclitaxel followed by dose-dense doxorubicin and cyclophosphamide on pathologic complete response rates in stage II to III triple-negative breast cancer: CALGB 40603 (A). J Clin Oncol 33(1):13-21

13. Von Minckwitz G, Schneeweiss A, Loibl S, Salat C, Denkert C, Rezai $M$ et al (2014) Neoadjuvant carboplatin in patients with triple-negative and HER2-positive early breast cancer (GeparSixto; GBG 66): a randomised phase 2 trial. Lancet Oncol 15(7):747-756

14. Carey LA, Dees EC, Sawyer L, Gatti L, Moore DT, Collichio $F$ et al (2007) The triple negative paradox: primary tumor chemosensitivity of breast cancer subtypes. Clin Cancer Res 13(8):2329-2334

15. Cortazar P, Zhang L, Untch M, Mehta K, Costantino JP, Wolmark N et al (2014) Pathological complete response and long-term clinical benefit in breast cancer: the CTNeoBC pooled analysis. Lancet 384(9938):164-172

16. Lehmann BD, Jovanović B, Chen X, Estrada MV, Johnson KN, Shyr Y et al (2016) Refinement of triple-negative breast cancer molecular subtypes: implications for neoadjuvant chemotherapy selection. PLoS ONE 11(6):e0157368

17. Lehmann BD, Bauer JA, Chen X, Sanders ME, Chakravarthy AB, Shyr Y et al (2011) Identification of human triple-negative breast cancer subtypes and preclinical models for selection of targeted therapies. J Clin Invest 121(7):2750-2767

18. Lehmann BD, Pietenpol JA (2014) Identification and use of biomarkers in treatment strategies for triple-negative breast cancer subtypes. J Pathol 232(2):142-150

19. Burstein MD, Tsimelzon A, Poage GM, Covington KR, Contreras A, Fuqua SAW et al (2015) Comprehensive genomic analysis identifies novel subtypes and targets of triple-negative breast cancer. Clin Cancer Res 21(7):1688-1698
20. Koboldt DC, Fulton RS, McLellan MD, Schmidt H, KalickiVeizer J, McMichael JF et al (2012) Comprehensive molecular portraits of human breast tumours. Nature 490(7418):61-70

21. Shah SP, Roth A, Goya R, Oloumi A, Ha G, Zhao Y et al (2012) The clonal and mutational evolution spectrum of primary triplenegative breast cancers. Nature 486(7403):395-399

22. Turner N, Tutt A, Ashworth A (2004) Hallmarks of "BRCAness" in sporadic cancers. Nat Rev Cancer 4(10):814-819

23. Lips EH, Mulder L, Oonk A, Van Der Kolk LE, Hogervorst FBL, Imholz ALT et al (2013) Triple-negative breast cancer: BRCAness and concordance of clinical features with BRCA1-mutation carriers. Br J Cancer 108(10):2172-2177

24. Tutt A, Ellis P, Kilburn L, Gilett C, Pinder S, Abraham J et al (2014) The Tnt Trial: a randomised phase Iii trial of Carboplatin (C) Compared with Docetaxel (D) for patients with metastatic or recurrent locally advanced triple negative or Brca1/2 breast cancer (Cruk/07/012) Abstr S3-01. In: San Antonio Breast Cancer Symposium. San Antonio, TX

25. Rugo HS, Olopade OI, DeMichele A, Yau C, Van't Veer LJ, Buxton MB et al (2016) Adaptive randomization of veliparib-carboplatin treatment in breast cancer. N Engl J Med 375(1):23-34

26. Ando M, Yamauchi H, Aogi K, Shimizu S, Iwata H, Masuda $\mathrm{N}$ et al (2014) Randomized phase II study of weekly paclitaxel with and without carboplatin followed by cyclophosphamide/ epirubicin/5-fluorouracil as neoadjuvant chemotherapy for stage II/IIIA breast cancer without HER2 overexpression. Breast Cancer Res Treat 145(2):401-409

27. Chang HR, Glaspy J, Allison MA, Kass FC, Elashoff R, Chung DU et al (2010) Differential response of triple-negative breast cancer to a docetaxel and carboplatin-based neoadjuvant treatment. Cancer 116(18):4227-4237

28. Guarneri V, Dieci MV, Bisagni G, Boni C, Cagossi K, Puglisi F et al (2015) Preoperative carboplatin-paclitaxel-bevacizumab in triple-negative breast cancer: final results of the phase II Ca.Pa. Be study. Ann Surg Oncol 22(9):2881-2887

29. Fan Y, Xu BH, Yuan P, Ma F, Wang JY, Ding XY et al (2013) Docetaxel-cisplatin might be superior to docetaxel-capecitabine in the first-line treatment of metastatic triple-negative breast cancer. Ann Oncol 24(5):1219-1225

30. Abramson VG, Lehmann BD, Ballinger TJ, Pietenpol JA (2015) Subtyping of triple-negative breast cancer: implications for therapy. Cancer 121(1):8-16

31. Stover DG, Winer EP (2015) Tailoring adjuvant chemotherapy regimens for patients with triple negative breast cancer. Breast 24:S132-S135

32. Harvey JM, Clark GM, Osborne CK, Allred DC (1999) Estrogen receptor status by immunohistochemistry is superior to the ligand-binding assay for predicting response to adjuvant endocrine therapy in breast cancer. J Clin Oncol 17(5):1474

33. Elston CW, Ellis IO (1991) Pathological prognostic factors in breast cancer. The value of histological grade in breast cancer: experience from a large study with long-term follow-up. Histopathology 19:403-410

34. Ellis I, Al-Sam S, Anderson N, Carder P, Deb R, Girling A et al (2016) Pathology reporting of breast disease in surgical excision specimens incorporating the dataset for histological reporting of breast cancer. The Royal College of Pathologists, London

35. Moossdorff M, Van Roozendaal LM, Strobbe LJA, Aebi S, Cameron DA, Dixon JM et al (2014) Maastricht Delphi consensus on event definitions for classification of recurrence in breast cancer research. J Natl Cancer Inst. https://doi.org/10.1093/jnci/dju288

36. Wittekind C, Sobin L, Gospodarowicz M (2009) TNM classification of malignant tumours, 7th ed. Wiley, Hoboken

37. Provenzano E, Bossuyt V, Viale G, Cameron D, Badve S, Denkert $C$ et al (2015) Standardization of pathologic evaluation and reporting of postneoadjuvant specimens in clinical trials of breast 
cancer: recommendations from an international working group. Mod Pathol 28(9):1185-1201

38. Hammond ME, Hayes DF, Dowsett M, Allred DC, Hagerty KL, Badve S et al (2010) American Society of Clinical Oncology/ College of American Pathologists guideline recommendations for immunohistochemical testing of estrogen and progesterone receptors in breast cancer (unabridged version). Arch Pathol Lab Med 134(7):48-72

39. Wolff AC, Hammond MEH, Hicks DG, Dowsett M, McShane LM, Allison KH et al (2013) Recommendations for human epidermal growth factor receptor 2 testing in breast. J Clin Oncol 31(31):3997-4013

40. Fisher B, Brown A, Mamounas E, Wieand S, Robidoux A, Margolese RG et al (1997) Effect of preoperative chemotherapy on local-regional disease in women with operable breast cancer: findings from National Surgical Adjuvant Breast and Bowel Project B-18. J Clin Oncol 15(7):2483-2493

41. Bonadonna G, Valagussa P, Brambilla C, Ferrari L, Moliterni A, Terenziani M et al (1998) Primary chemotherapy in operable breast cancer: eight-year experience at the Milan Cancer Institute. J Clin Oncol 16(1):93-100

42. Fisher B, Bryant J, Wolmark N, Mamounas E, Brown A, Fisher ER et al (1998) Effect of preoperative chemotherapy on the outcome of women with operable breast cancer. J Clin Oncol 16(8):2672-2685

43. Bear HD, Anderson S, Brown A, Smith R, Mamounas EP, Fisher B et al (2003) The effect on tumor response of adding sequential preoperative docetaxel to preoperative doxorubicin and cyclophosphamide: preliminary results from national surgical adjuvant breast and bowel project protocol B-27. J Clin Oncol 21(22):4165-4174

44. Berruti A, Amoroso V, Gallo F, Bertaglia V, Simoncini E, Pedersini R et al (2014) Pathologic complete response as a potential surrogate for the clinical outcome in patients with breast cancer after neoadjuvant therapy: a meta-regression of 29 randomized prospective studies. J Clin Oncol 32(34):3883-3891

45. Rastogi P, Anderson SJ, Bear HD, Geyer CE, Kahlenberg MS, Robidoux A et al (2008) Preoperative chemotherapy: updates of national surgical adjuvant breast and bowel project protocols B-18 and B-27. J Clin Oncol 26(5):778-785

46. Hatzis C, Symmans WF, Zhang Y, Gould RE, Moulder SL, Hunt KK et al (2016) Relationship between complete pathologic response to neoadjuvant chemotherapy and survival in triplenegative breast cancer. Clin Cancer Res 22(1):26-33

47. Pusztai L, Szekely B, Hatzis C (2017) Is complete response the answer? Ann Oncol 28(8):1681-1683

48. Alba E, Chacon JI, Lluch A, Anton A, Estevez L, Cirauqui B et al (2012) A randomized phase II trial of platinum salts in basal-like breast cancer patients in the neoadjuvant setting. Results from the GEICAM/2006-03, multicenter study. Breast Cancer Res Treat 136(2):487-493

49. Breast Cancer Version 2 [Internet] (2017) https://www.nccn. org/professionals/physician_gls/pdf/breast.pdf

50. Vasey PA, Jayson GC, Gordon A, Gabra H, Coleman R, Atkinson R et al (2004) Phase III randomized trial of docetaxel-carboplatin versus paclitaxel-carboplatin as first-line chemotherpy for ovarian carcinoma. J Natl Cancer Inst 96(22):1682-1691

51. Perez EA, Hillman DW, Stella PJ, Krook JE, Hartmann LC, Fitch TR et al (2000) A phase II study of paclitaxel plus carboplatin as first-line chemotherapy for women with metastatic breast carcinoma. Cancer 88(1):124-131

52. Perez EA, Suman VJ, Fitch TR, Mailliard JA, Ingle JN, Cole JT et al (2005) A phase II trial of docetaxel and carboplatin as first-line chemotherapy for metastatic breast cancer: NCCTG study N9932. Oncology 69(2):117-121
53. Sharma P, López-Tarruella S, García-Saenz JA, Ward C, Connor CS, Gómez HL et al (2017) Efficacy of Neoadjuvant carboplatin plus docetaxel in triple-negative breast cancer: combined analysis of two cohorts. Clin Cancer Res 23(3):649-657

54. Zhao S, Ma D, Xiao Y, Jiang Y-Z, Shao Z-M (2018) Clinicopathologic features and prognoses of different histologic types of triple-negative breast cancer: a large population-based analysis. Eur J Surg Oncol 44(4):420-428

55. Liao H-Y, Zhang W-W, Sun J-Y, Li F-Y, He Z-Y, Wu S-G (2017) The clinicopathological features and survival outcomes of different histological subtypes in triple-negative breast cancer. J Cancer 9(2):296-303

56. Mills MN, Yang GQ, Oliver DE, Liveringhouse CL, Ahmed KA, Orman AG et al (2018) Histologic heterogeneity of triple negative breast cancer: a National Cancer Centre Database analysis. Eur J Cancer [Internet] 98:48-58

57. Urru SAM, Gallus S, Bosetti C, Moi T, Medda R, Sollai E et al (2018) Clinical and pathological factors influencing survival in a large cohort of triple-negative breast cancer patients. BMC Cancer 18(1):56

58. Masuda H, Baggerly KA, Wang Y, Zhang Y, Gonzalez-Angulo AM, Meric-Bernstam F et al (2013) Differential response to neoadjuvant chemotherapy among 7 triple-negative breast cancer molecular subtypes. Clin Cancer Res 19(19):5533-5540

59. Nielsen TO, Hsu FD, Jensen K, Cheang M, Karaca G, Hu Z et al (2004) Immunohistochemical and clinical characterization of the basal-like subtype of invasive breast carcinoma. Clin Cancer Res 10(16):5367-5374

60. Rakha EA, El-Sayed ME, Green AR, Lee AHS, Robertson JF, Ellis IO (2007) Prognostic markers in triple-negative breast cancer. Cancer 109(1):25-32

61. Foulkes WD, Grainge MJ, Rakha EA, Green AR, Ellis IO (2009) Tumor size is an unreliable predictor of prognosis in basal-like breast cancers and does not correlate closely with lymph node status. Breast Cancer Res Treat 117(1):199-204

62. Abdulkarim BS, Cuartero J, Hanson J, Deschênes J, Lesniak D, Sabri S (2011) Increased risk of locoregional recurrence for women with T1-2N0 triple-negative breast cancer treated with modified radical mastectomy without adjuvant radiation therapy compared with breast-conserving therapy. J Clin Oncol 29(21):2852-2858

63. Adkins FC, Gonzalez-Angulo AM, Lei X, Hernandez-Aya LF, Mittendorf EA, Litton JK et al (2011) Triple-negative breast cancer is not a contraindication for breast conservation. Ann Surg Oncol 18(11):3164-3173

64. Voduc KD, Cheang MCU, Tyldesley S, Gelmon K, Nielsen TO, Kennecke H (2010) Breast cancer subtypes and the risk of local and regional relapse. J Clin Oncol 28(10):1684-1691

65. Cameron D, Brown J, Dent R, Jackisch C, Mackey J, Pivot X et al (2013) Adjuvant bevacizumab-containing therapy in triple-negative breast cancer (BEATRICE): primary results of a randomised, phase 3 trial. Lancet Oncol 14(10):933-942

66. Gerber B, Loibl S, Eidtmann H, Rezai M, Fasching PA, Tesch H et al (2013) Neoadjuvant bevacizumab and anthracycline-taxanebased chemotherapy in 678 triple-negative primary breast cancers; results from the geparquinto study (GBG 44). Ann Oncol 24(12):2978-2984

67. Bear HD, Tang G, Rastogi P, Geyer CE, Liu Q, Robidoux A et al (2015) Neoadjuvant plus adjuvant bevacizumab in early breast cancer (NSABP B-40 [NRG Oncology]): secondary outcomes of a phase 3, randomised controlled trial. Lancet Oncol 16(9): 1037-1048

68. Sataloff DM, Mason BA, Prestipino AJ, Seinige UL, Lieber CP, Baloch Z (1995) Pathologic response to induction chemotherapy in locally advanced carcinoma of the breast: a determinant of outcome. J Am Coll Surg 180(3):297-304 
69. Symmans WF, Peintinger F, Hatzis C, Rajan R, Kuerer H, Valero $\mathrm{V}$ et al (2007) Measurement of residual breast cancer burden to predict survival after neoadjuvant chemotherapy. J Clin Oncol 25(28):4414-4422

70. Symmans WF, Wei C, Gould R, Yu X, Zhang Y, Liu M et al (2017) Long-term prognostic risk after neoadjuvant chemotherapy associated with residual cancer burden and breast cancer subtype. J Clin Oncol 35(10):1049-1060

71. Peintinger F, Sinn B, Hatzis C, Albarracin C, Downs-Kelly E, Morkowski J et al (2015) Reproducibility of residual cancer burden for prognostic assessment of breast cancer after neoadjuvant chemotherapy. Mod Pathol 28(7):913-920
72. Iwamoto T, Booser D, Valero V, Murray JL, Koenig K, Esteva FJ et al (2012) Estrogen receptor (ER) mRNA and ER-related gene expression in breast cancers that are 1-10\% ER-positive by immunohistochemistry. J Clin Oncol 30(7):729-734

73. Sheffield BS, Kos Z, Asleh-Aburaya K, Wang XQ, Leung S, Gao $D$ et al (2016) Molecular subtype profiling of invasive breast cancers weakly positive for estrogen receptor. Breast Cancer Res Treat 155(3):483-490

74. Liedtke C, Rody A (2015) New treatment strategies for patients with triple-negative breast cancer. Curr Opin Obstet Gynecol 27(1):77-84

\section{Affiliations}

\section{Elaine M. Walsh ${ }^{1,2}$ - Aliaa Shalaby ${ }^{1}$ - Mark O'Loughlin ${ }^{1} \cdot$ Nessa Keane $^{1} \cdot$ Mark J Webber $^{1} \cdot$ Michael J. Kerin $^{3}$. Maccon M. Keane ${ }^{2}$. Sharon A. Glynn ${ }^{1}$. Grace M. Callagy ${ }^{1}{ }^{\mathbb{C}}$}

Elaine M. Walsh

elaine1walsh@gmail.com; ewalsh13@jhmi.edu

Aliaa Shalaby

aliaashalaby@gmail.com

Mark O’Loughlin

mark.oloughlin@hse.ie

Nessa Keane

nessakeane07@gmail.com

Mark J Webber

mark.webber@nuigalway.ie

Michael J. Kerin

michael.kerin@nuigalway.ie
Maccon M. Keane

maccon.keane@hse.ie

Sharon A. Glynn

sharon.glynn@nuigalway.ie

1 Discipline of Pathology, Lambe Institute for Translational Research, NUI Galway, Costello Road, Galway, Ireland

2 Department of Medical Oncology, University Hospital Galway, Galway, Ireland

3 Discipline of Surgery, Lambe Institute for Translational Research, NUI Galway, Costello Road, Galway, Ireland 